\title{
reCHERches
}

Culture et histoire dans l'espace roman

$12 \mid 2014$

Empreintes/emprunts : entre forces de conformisation et forces d'innovation

\section{Un planctus avant-gardiste : Llanto por Ignacio Sánchez Mejías de F. García Lorca}

\section{Carole Egger}

\section{(2) OpenEdition}

\section{Journals}

Édition électronique

URL : https://journals.openedition.org/cher/7110

ISSN : 2803-5992

\section{Éditeur}

Presses universitaires de Strasbourg

\section{Édition imprimée}

Date de publication : 7 juillet 2014

Pagination : $211-222$

ISBN : 978-2-86820-569-8

ISSN : 1968-035X

\section{Référence électronique}

Carole Egger, « Un planctus avant-gardiste : Llanto por Ignacio Sánchez Mejías de F. García Lorca », reCHERches [En ligne], 12 | 2014, mis en ligne le 13 décembre 2021, consulté le 15 décembre 2021. URL : http://journals.openedition.org/cher/7110

\section{c) (i) (2)}

Ce(tte) œuvre est mise à disposition selon les termes de la Licence Creative Commons Attribution Pas d'Utilisation Commerciale - Partage dans les Mêmes Conditions 4.0 International. 


\title{
Un planctus avant-gardiste: Llanto por Ignacio Sánchez Mejías de F. García Lorca
}

Carole Egger

CHER Strasbourg

\begin{abstract}
$F^{l}$ llanto por Ignacio Sánchez Mejías a été considéré comme un sommet Cdans la poésie de García Lorca, son œuvre la plus achevée. Lorsqu'il l'écrit, en 1934, l'art de Lorca a atteint sa pleine maturité et le poème reflète l'art consommé avec lequel le poète fonde dans un même creuset références religieuses et profanes, tradition savante et recréation populaire, formes et images conventionnelles et singulièrement originales et novatrices. C'est ainsi qu'en parle son frère Francisco García Lorca, dans son livre posthume:
\end{abstract}

La razón de su valor radica en que el poeta, ya en la madurez de su técnica, refleja con una gran libertad de factura muchos de sus procedimientos típicos y de sus recursos poéticos. Es un poema de integración y por ello, en cierto modo, el más lorquiano, el que refleja mejor, el rostro del poeta. En él alternan innovación y tradición, libertad creadora y disciplina, ímpetu lírico y enfrenamiento ${ }^{1}$.

C'est sur les antagonismes contenus dans cette dernière affirmation que je m'arrêterai afin d'inventorier et d'analyser, selon le programme de ce colloque, les forces de "conformisation" et d'innovation qui font de cette tétralogie élégiaque le chef d'œuvre du poète andalou. Je m'attacherai notamment à montrer que cette tension structurelle entre passé et présent, entre texte et intertexte concerne aussi bien le contenu sémantique que la forme strophique et métrique des quatre poèmes.

1 García Lorca Francisco, 1980, Federico y su mundo, Madrid, Alianza Editorial.

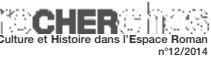


Lorca choisit de nommer sa composition llanto et non pas élégie, sans doute parce que le terme permet de convoquer celui de planto, le planctus médiéval - hérité des thrènes de la Grèce antique - qui se définit comme un genre lyrique des lettres latines qui apparaît au viII ${ }^{\mathrm{e}}$ siècle et se maintiendra jusqu'au XIII siècle, date à laquelle il passera dans la langue vernaculaire. Bien avant les fameuses Coplas a la muerte de su padre Jorge Manrique qui s'inscrivent dans la même veine de l'éloge funèbre, le planctus Rex obit pour ne prendre qu'un exemple - entre autres textes de lamentations, de deploratio, d'oraisons funèbres et d'élégies -, rendait hommage à l'un des plus importants souverains de Castille, Alphonse VIII le Noble, fondateur du monastère de las Huelgas où fut consigné le fameux manuscrit compilé au début du XIve siècle, connu sous le nom de Codex de las huelgas ${ }^{2}$. Pour rendre hommage à son ami et toreador Sanchez Mejías le planctus présente en fait plusieurs avantages. En premier lieu, le respect de certains éléments de sa composition permet au poète de s'inscrire dans une tradition poétique et lyrique, hispanique certes mais qui, débordant aussi largement les frontières de la péninsule, donne à leur terre andalouse commune un caractère universel. Ensuite, le planctus est destiné à être chanté et Le Codex de las huelgas comme le Llanto... de Lorca n'ont pas fini d'inspirer musicologues et musiciens ${ }^{3}$. Enfin, comme le genre n'a pas de structure propre ni de longueur déterminée, Lorca va composer selon un rythme, une prosodie et des mélanges strophiques et métriques tout à fait originaux et singuliers, un long poème de deux cents vingt-deux vers qui renoue avec le canon élégiaque de grande ampleur.

Il ne s'agit pas de proposer ici une énième version de commentaire du texte, mais de pointer les principaux éléments qui participent de cette alliance indéfectible entre passé et présent. J'examinerai en premier lieu tous les procédés qui contribuent à inscrire la composition dans un processus de sacralisation mythique: célébration d'un rituel, abondance de références appartenant au mythe, musique omniprésente qui prend parfois des accents liturgiques. Dans le même temps, je signalerai les éléments qui renvoient

2 Fondé en 1184 par le roi de Castille Alphonse VIII, le monastère de moniales cisterciennes de Las Huelgas de Burgos devint sous Alphonse X un centre culturel important où juifs, musulmans et chrétiens cohabitaient harmonieusement. La musique en particulier s'y développa jusqu'à avoir à son service cent moniales choristes. C'est au début du XIII ${ }^{\mathrm{e}}$ siècle que fut copié le Codex de las Huelgas, qui comprend plus de 180 pièces liturgiques.

3 Il existe une multitude de versions du Llanto... mis en musique depuis une des toutes premières versions, celle de Maurice Ohana avec Maria Casares comme récitante. Il y a encore quelques années, Vicente Pradal créait un oratorio à partir de ce texte. 
au respect, mais aussi à la subversion, de la tradition élégiaque. Enfin je m'attacherai à montrer que si le rythme est parfois empreint de solennité religieuse, le traitement métrique et métaphorique est le plus souvent totalement inédit.

La composition poétique s'inscrit dans une double tradition, celle, bien sûr, qui consiste à pleurer les morts mais aussi celle qui consiste en une célébration de la fête taurine.

[...] el toreo, es probablemente la riqueza poética y vital mayor de España, increíblemente desaprovechada por los escritores y artistas, debido principalmente a una falsa educación pedagógica que nos han dado y que hemos sido los hombres de mi generación los primeros en rechazar. Creo que los toros es la fiesta más culta que hay hoy en el mundo; es el drama puro, en el cual el español derrama sus mejores lágrimas y sus mejores bilis. Es el único sitio adonde se va con la seguridad de ver la muerte rodeada de la más deslumbradora belleza. ${ }^{4}$

La structure rituelle, de cérémonie sacrificielle que possède le poème a été soulignée par Gustavo Correa dans son livre sur La poésie mythique chez García Lorca ${ }^{5}$. Déjà l'annulation du temps à laquelle procède la réitération temporelle "à cinq heures du soir...» dans le premier poème de la tétralogie, La cogida y la muerte, la réitération du refrain après chacune des images créées, centre tout le poème sur l'implacable circonstance temporelle. Le poème ne se déroule pas, il ne s'énonce pas dans une temporalité réaliste, il reste suspendu à cette heure fatidique qui arrête le temps. Et chaque image est ramenée à ce moment terriblement dramatique, ou plutôt à cet instant où se rejoignent l'être et le non être. La simultanéité de toutes les images est encore soulignée par l'absence de verbes et l'abondance des phrases nominales, aux vers 5, 15, 19, 21, 23, 43 et 50, ou bien encore par l'adverbe de temps "ya», aux vers 5, 13, 37 et 41, qui réduit encore, si besoin était, la distance temporelle entre les actions pour les ramener à cet instant fatidique précis, juste cinq heures du soir.

Puis, un enfant, sorte de héraut enfantin, d'ange de la mort, amène le drap funéraire, le suaire, et on indique que la tombe est déjà préparée (vers 5 " una espuerta de cal ya prevenida»); le couffin de chaux blanche n'est autre que celui que les fossoyeurs tiennent préparé au moment de l'enterrement et la séquence a lieu face au chœur tribal (vers 21 «en las esquinas grupos

4 Federico García Lorca «Diálogos de un caricaturista salvaje» in Obras Completas, tomo II, Madrid, Aguilar, 1980, p. 1128.

5 Gustavo Correa, 1975, La poesía mítica de Federico García Lorca, Madrid, Gredos. 
de silencio»). Le ya dans l'expression "ya prevenida», de même que le vers suivant: (vers 7) «Lo demás era muerte y sólo muerte», renvoient sans doute au contexte particulièrement tragique qui avait entouré la mort d'Ignacio ${ }^{6}$.

On a ainsi une répétition énoncée par la voix poétique dans une sorte d'hébétude, comme poussée par la nécessité de verbaliser l'inconcevable. Le poète est comme assommé par la nouvelle qui s'est d'ailleurs peut-être répandue selon cette même formulation, en particulier dans la presse "Aujourd'hui, à cinq heures du soir, dans les arènes de Manzanares, Ignacio Sánchez Mejías fut gravement blessé par un taureau appartenant à l'élevage de Ayala et qui avait pour nom Granadino». On retrouve à la fois l'expression de cette fulgurante surprise causée par la mort, de même que la dimension collective de ce llanto qui est l'expression de ces "grupos de silencio", "groupes de silence» du vers 21 dans le planctus médiéval que j'ai pris pour exemple: "assi llago los coraçones de todos como si los firiesse saeta ", comme si une flèche avait percé le cœur de tous - dit le texte ancien. Le vers 39 , un des plus beaux du poème «el cuarto se irisaba de agonía», décrit le couchant, - c'est-à-dire selon une image topique, la fin de vie d'Ignacio -, comme une agonie qui prend possession de l'espace de la chambre comme les couleurs de l'arc-en-ciel celui du ciel.

Comme l'a fait remarquer Angel del Río7, les quatre parties de ce chant funèbre pour Ignacio Sánchez Mejías s'organisent et s'enchaînent selon un certain rythme musical, un peu à la façon des quatre temps d'une sonate. Ce rythme musical se concrétise dans le poème par des modèles métriques très différents d'une partie à l'autre et qui possèdent eux-mêmes des modulations internes très précises.

Dans La cogida y la muerte, on assiste au fatal et tragique événement, au coup de corne brutal, la cogida, - mal traduite par «la blessure» qui ne dit pas la violence de l'action - et à l'agonie du toréador. L'indication temporelle, devenue célébrissime, qui scande le poème «a las cinco de la tarde » apparaît vingt cinq fois, plus cinq avec des variantes, ce qui apparente le vers aux anciens motets, ces chants d'église à plusieurs voix de l'époque médiévale. La critique a par ailleurs fréquemment évoqué la conception

6 La mort d'Ignacio en effet apparaît un peu comme la mort annoncée. Ignacio ne devait pas toréer ce jour-là, plusieurs événements catastrophiques dans les jours précédents pouvaient être interprétés comme signes prémonitoires du destin funeste du toréador. Et c'est bien ainsi que l'entendit Lorca, lequel était en outre assez superstitieux.

7 Angel del Río, 1952, Vida y obras de Federico García Lorca, Heraldo de Aragón, Zaragoza. Cité par Miguel García Posada, 1979, Federico García Lorca, Madrid, escritores de todos los tiempos, EDAF. 
liturgique que Lorca avait de la corrida. C'est en outre l'octosyllabe qui est ici associé à l'hendécasyllabe, et non l'heptasyllabe comme c'est souvent le cas dans la tradition lyrique hispanique. Le chiffre, si fréquemment utilisé dans la poésie de Lorca, ici le chiffre cinq, reviendra près de soixante fois dans tout le livre.

Dans le premier poème, le moi poétique n'était pas nommé, le toréador non plus. Il y avait juste une volonté manifeste d'objectiver la mort, de manière impersonnelle, de façon à la figer dans un instant précis, cinq heures du soir et lui donner ainsi un caractère exemplaire. Dans le deuxième poème de la tétralogie au contraire, Lorca laisse libre cours à sa subjectivité, il exprime ses propres sentiments; ce lyrisme en fait aussi le moment le plus émouvant. La sangre derramada, Le sang versé ou répandu, est dominé par l'octosyllabe et l'hendécasyllabe, assonancés en e-a aux vers pairs. C'est là qu'apparait la lune, symbole de mort dans la mythologie lorquienne: "La luna de par en par / Caballo de nubes quietas» (vers. 58-59). Elle répand la mort de toutes parts, telle une messagère inquiétante et hiératique, qui répand aussi sa lumière grisâtre sur la plaza dont rêve ou dont a rêvé Ignacio Sánchez Mejías et qui porte la marque des "sauces", ces "árboles de lágrimas", ces arbres pleureurs que l'on retrouvera dans le poème suivant Cuerpo presente, au vers 151. Cette lumière mortifère est nécessaire au poète pour éviter que le soleil d'août ne brûle son souvenir.

À partir du vers 67 le poème prend une configuration nettement mythique avec l'assimilation très ancienne de la vache et de la lune puisqu'il semble qu'il s'agisse là d'une alliance que l'on trouve déjà chez les sumériens: «la nuit étoilée est dominée par le taureau prestigieux dont la vache féconde est la pleine lune, dont le troupeau est la voie lactée»8.

Le sang s'énonce désormais au pluriel, il semble avoir fécondé la terre, les taureaux se sont multipliés, ils deviendront au vers 100 les taureaux célestes des ganaderías (vers 98) et même, les taureaux de pierre, ceux de Guisando9, qui sont rendus à une presque vie pour mugir et gémir sur leurs deux siècles chrétiens d'existence:

Y los toros de Guisando

Casi muerte y casi piedra

Mugieron como dos siglos

Hartos de pisar la tierra (vers 70-74)

8 Jean Chevalier et Alain Gheerbrant, 1997, Dictionnaire des symboles, Paris, Laffont, p. 988.

9 Statues de pierre - datant de l'époque romaine - encore visibles dans la province de Ávila. 
Le passage du monde taurin au monde lunaire dote cette mort d'une dimension cosmique et les constellations vont participer à la diffusion de la nouvelle: «Hubo un aire de voces secretas / Que gritaban a toros celestes / Mayorales de pálida niebla» (vers 98 à 101), vers où l'on reconnaîtra l'influence gongorina.

La sangre derramada équivaut à une cérémonie du sang versé, le poète refuse de voir le sang de son ami sur l'arène stérile. Ce sang versé, sang répandu, deviendra "sangre abierta», sang ouvert (vers. 84) puis «sangre dura », (vers. 136), dur, il est la matérialisation de la mort concrète que Lorca ne voudra jamais voir ${ }^{10}$. Le spectre d'Ignacio monte les marches de la place dans une atmosphère onirique, telle une victime sacrificielle. Puis, le poète fait l'éloge du héros dans une veine proche du pregón de alabanzas médiéval, que l'on retrouve par exemple dans le planctus à la mort d'Alphonse VIII le Noble.

Le panégyrique du défunt "No hubo príncipe en Sevilla», vers suivi d'une série d'exclamations:

¡Qué gran torero en la plaza!

¡Qué buen serrano en la sierra!

¿Qué blando con las espigas!

¡Qué duro con las espuelas!

¡Qué tierno con el rocío!

¡Qué deslumbrante en la feria!

¡Qué tremendo con las últimas

banderillas de tiniebla! (vers 114-121)

rappellentégalementlesvers de Jorge Manrique: «Quéenemigo d’enemigos!/ ¡Qué maestro d'esforçados e valientes!». Mais ici, la réputation du héros ne lui assurera pas la gloire éternelle, comme dans les élégies classiques. Finalement, Ignacio devient une sorte de nouvelle figure christique, dont le sang court, illimité "Que no hay cáliz que lo contenga» «car il n’y a pas de calice pour le contenir» (vers 140).

Cette identification christique invite néanmoins à une interprétation un peu particulière quand on sait que Lorca considérait inutile la mort du Christ, autre mort violente, et qu'il a identifié le chœur tribal au public, dans l'œuvre qui porte ce nom, un public avide de mort. C'est pourquoi lorsque

10 Lorca ne fera pas le voyage jusqu' à Manzanares, il n'ira pas non plus à Madrid quand Ignacio y est transféré, il attendra par contre patiemment chez lui des nouvelles jusqu'à l'annonce de sa fin. Il ne suivra pas la Barraca au soir du 12 août à Santander où le groupe était attendu pour une représentation, il dira finalement à ses amis qu'il ne veut pas voir le cadavre de son ami. 
Ignacio agonise «el gentío rompía las ventanas» (vers 47) et lorsque la victime monte les marches, le poète parle avec horreur de:

ese chorro que ilumina

los tendidos y se vuelca

sobre la pana y el cuero

de muchedumbre sedienta. (vers. 88-91)

Ce rituel revêt un sens singulier, le poète ne veut pas voir ce sang répandu, mais il ne s'agit pas seulement d'un geste instinctif d'horreur. Comme l'a si bien dit Miguel García Posada, le poète «repousse le sacrifice de la vie comme thérapeutique de salut»; la mort ne conduit qu'à la mort et non comme dans la mythologie chrétienne, au salut. C'est pourquoi le poète écarte avec violence toute possibilité de consolation ou d'auto-tromperie: « ¿Quién arruga el sudario? No es verdad lo que dice.» au vers 171 de Cuerpo presente.

Les références à l'Antiquité: "Aire de Roma andaluza/ Le doraba la cabeza» (vers. 110-111) accusent la grandeur et la beauté du héros qui n'a pas fermé les yeux face au danger: "No se cerraron sus ojos/ Cuando vio los cuernos cerca» (vers. 94-95). Il s'agit là bien évidemment de rendre hommage au courage légendaire de Sanchez Mejías dont certains ont pu penser, face aux risques inconsidérés qu'il avait coutume de prendre, qu'en définitive il souhaitait plus ou moins inconsciemment mourir dans l'arène. Les mères évoquées au vers 94: "pero las madres terribles levantaron la cabeza», ce sont Les Parques qui donnent leur assentiment, les déesses mythologiques sont ici l'expression de la fatalité. Finalement, c'est le ciel tout entier qui s'accorde avec cette mort qui est comme inscrite dans les étoiles.

Après le cri déchirant de La sangre derramada, dans Cuerpo presente, la voix du poète se fait à la fois plus libre - on a ici toute une série d'associations subconscientes ou semi-conscientes - et à la fois plus mesurée. Cuerpo presente, corps présent ou bien présence du corps, cette troisième partie se compose de 49 alexandrins, vers propre au Mester de clerecía et à la cuaderna vía du Libro de Alexandre, de Gonzalo de Berceo ou du Libro de buen amor où nous trouvons par ailleurs un très beau planctus à la mort de l'entremetteuse Trotaconventos. Sauf qu'ici, nous n'avons pas de tetrásforo monorrimo mais des quatrains à rime libre. Sur la pierre, où gît Ignacio, le bien né, - tel El Cid Campeador « el que en buen hora nació » - son corps de " capitaine» "atado por la muerte» commence à s'effacer, à s'estomper. Et la méditation invite à considérer la dimension cosmique de cette mort. 
On a de fait un curieux contraste entre une très grande liberté créatrice d'une part - on a parfois le sentiment d'avoir affaire à un poème surréaliste et une certaine tempérance qui s'exprime dans une sorte de réserve formelle; c'est par exemple la seule partie du poème qui soit composée du même mètre, l'alexandrin. Autrement dit, c'est au moment où la structure métrique devient la plus rigide que les images se font plus libres, surréalistes: «La piedra es una frente donde los sueños gimen/ Sin tener agua curva ni cipreses helados» (vers. 148-149). La froideur de la pierre a anéanti la possibilité pour le front de continuer à rêver (souvenons-nous du vers 37 « El toro ya mugía por su frente») et les songes eux-mêmes deviennent plainte " ils gémissent», ils sont sans vie, "sin tener agua curva», ce qui peut renvoyer au «surtidor», au «chorro», au jet d'eau, qui juste avant était jet de sang. Ils sont également sans mort, "ni cipreses helados», ils s'acheminent donc vers l'au-delà du néant.

Dans Cuerpo presente, le processus de sacralisation du profane qui oriente tout le poème se poursuit. On y trouve d'autres symboles comme celui de la pluie qui fertilise, de la vache nourricière et surtout, l'image du minotaure à travers laquelle Ignacio fait corps avec le taureau, participe de ce monde taurin céleste qui s'inscrit, on l'a vu, dans des mythologies très anciennes: «La muerte le ha cubierto de pálidos azufres / Y le ha puesto cabeza de oscuro minotauro» (vers. 161-162).

Cette métamorphose permet au poète de procéder au topoi de la description du corps, passage obligé du planctus, qui est ici cadavre, silence et puanteur:

Un silencio con hedores reposa

Estamos con un cuerpo presente que se esfuma

Con una forma clara que tuvo ruiseñores

$\mathrm{Y}$ la vemos llenarse de agujeros sin fondo.

(vers 167-170)

La présence du corps s'estompe, cette «forma clara», nous la retrouverons à la fin dans «un andaluz tan claro » et l'expression «tuvo ruiseñores» était déjà présente dans le deuxième poème où «la sangre», le sang était «ruiseñor de sus venas", le flux qui faisait chanter la vie en lui. Il y a ici un mélange de clarté et d'obscurité, d'abstrait et de concret. Si le monde poétique de Lorca possède cette extraordinaire unité, c'est parce que le poète crée tout un système de résonances qui rend cohérentes les choses les plus vagues ou les plus confuses et qui finalement éclaire le mystère, comme celui de cette étroite relation abstrait/ concret qui est une des marques de sa poésie. 
Le poète construit lui-même les propres repères de son monde poétique, il élabore lui-même sa propre cohérence.

Dans Alma ausente (Âme absente), hendécasyllabes, ennéasyllabes et alexandrins libres ne se conforment pas non plus à un schéma poétique conventionnel mais obéissent néanmoins à une structure formelle, soigneusement élaborée. Le poème sert d'abord la contemplation de l'irrémédiable, l'inscription de l'absence, corps et âme, de son ami défunt dans chacun des éléments qui constituaient son environnement familier. Les strophes sont des quatrains, exceptée la cinquième, qui compte cinq vers car le poème comporte deux mouvements. Le premier, composé de quatre quatrains, dit la disparition et l'absence à travers une série d'images:

No te conoce el toro ni la higuera

Ni caballos ni hormigas de tu casa.

No te conoce el niño ni la tarde

Porque te has muerto para siempre (vers 196-199)

selon un rythme quasi-psalmodié.

«Ni le taureau ni le figuier ne te connaissent» dit le texte espagnol et la traduction d'André Belamich dans la Pléiade ${ }^{11}$ explicite: «Tu n'es plus rien pour le figuier et le taureau». En outre «conocer» en castillan a également le sens de reconnaître. Les choses et les êtres de ton environnement familier ne te connaissent ni te reconnaissent, répète le poète. Si la conscience de l'homme se forge effectivement dans le regard de l'Autre, le poème dit qu'avec la mort il n'y a plus de regard, il n'y a plus de miroir où l'on puisse se reconnaître, où l'on puisse prendre vie. Aucun des éléments de ta vie passée n'est capable de te ramener à l'existence, ni la rappeler, car tu as perdu là l'essence même de ton être. Même cet ange de la mort que représentait cet enfant dans le premier poème, lors de ces cinq heures du soir funestes, ne te reconnaît pas parce "te has muerto para siempre", tu es mort pour toujours. On relèvera ici le paradoxe qui consiste d'une part à prolonger au moyen du passé composé l'instant de la mort dans le temps présent de l'énonciation et dans le même temps à l'inscrire dans l'éternité, "para siempre», comme si l'on pouvait mourir temporairement. La réitération de l'expression nous renvoie à l'hébétude initiale dont nous avons parlé, à cette difficulté, non pas seulement pour admettre, mais déjà simplement pour concevoir l'événement. Puis, progressivement, la mort acquiert, dans la quatrième strophe, une dimension universelle, sans que diminue jamais la

11 Federico García Lorca, 1990, Euvres complètes, tome II, André Belamich éd., Paris, Bibliothèque de la Pléiade ${ }^{\circ} 369$. 
force des images dans l'expression de ce qui est concret et tangible. Elle est mort universelle face à laquelle le poète nous invite à communier et l'image est celle d'une fosse commune, «un montón» où "perros sin vida», "perros apagados», nous nous entassons. La rupture métrique et strophique (la strophe comme le vers s'allongent) souligne le contraste entre l'absence du défunt et la présence puissante et vivante de la voix poétique qui s'impose alors avec force. À un espace dominé par le vide, la négation, le silence et l'annihilation progressive, le poète substitue les accents mélodiques de son chant. À travers des images métonymiques, énoncées chacune dans un vers qui correspond à une unité sémantique, le poète substitue à la prière des planctus d'autrefois, la prolongation de son chant en forme d'éloge où il loue l'élégance, la culture, la relation amoureuse qu'entretenait Ignacio avec la mort, tel un héros de la tragédie antique. Car l'oubli est inéluctable, comme la mort. Si jusque là Ignacio apparaissait tout au long du poème comme destinataire premier de la voix poétique, il disparaît désormais, y compris du poème.

Alma ausente dit également la disparition de toute référence religieuse ou mythologique. Ignacio ne se sauve pas, il meurt, il pourrit et disparaît de la surface de la terre en même temps qu'il disparaît du poème. Seul demeure le chant de Federico qui dit la perte, pour toute la communauté andalouse du fils bien aimé.

Dans tout le poème, la transposition poétique du réel à laquelle procède Lorca est comme dictée par une pensée en liberté qui fonctionne par un jeu d'associations et de mécanismes psychiques qui apparente le processus créatif à celui prôné à l'époque par les surréalistes. Mais chez Lorca, d'une part le référent est souvent identifiable et d'autre part, son imagination débridée demeure sous contrôle soit d'une forme strophique (le quatrain, le tercet, etc.), soit d'une forme métrique (octosyllabes, hendécasyllabes ou alexandrins), soit encore d'un genre (et on retrouve ici quelques uns des temps forts du planctus (la lamentation, l'éloge du défunt, la description physique du cadavre et, non pas la prière, comme dans le moule classique, mais le chant poétique final).

Je ne donnerai qu'un ou deux exemples de ce que j'ai appelé le référent poétique identifiable. Par exemple dans le premier poème, l'image allégorique de la mort se profilait, telle une femelle ovipare, pour venir déposer les œufs de sa progéniture au cœur de la blessure et s'assurer ainsi sa propre reproduction, ou prolifération: "La muerte puso huevos en la herida» (vers 29). Puis la danza de exequias, la danse de mort, présente dans 
la tradition médiévale, résonnait aux oreilles du mourant: «Huesos y flautas suenan en su oído» (vers. 35). Venait alors la gangrène, qui causa la mort d'Ignacio: «A lo lejos ya viene la gangrena» (vers. 41). Et un peu plus loin le vers énonce: «Trompas de lirio por las verdes ingles» (vers 43).

Eutimio Martín ${ }^{12}$ a donné une explication très prosaïque de cette dernière image, a priori très surréaliste. Comme il s'agit d'une gangrène gazeuse, les jambes enflent car des gaz se forment sous la peau, celle-ci devient jaunâtre (comme les trompes de la fleur d'iris) et l'aine se colore de vert à cause des ganglions dus à l'infection. On pourrait encore donner en exemple «Un ataud con ruedas es la cama» (vers 33) qui renvoie bien sûr au lit d'hôpital d'où Ignacio tomba à cause de la fièvre qui le faisait délirer.

D’autres images plus surréalistes demeurent plus ou moins hermétiques et renvoient, à n'en pas douter, à un imaginaire onirique et au monde des profondeurs intérieures. Parfois la liberté imaginative est jugulée par une structure syntaxique de type discursif logique, par exemple, à la troisième strophe de Cuerpo presente:

Porque la piedra coge simientes y nublados,

Esqueletos de alondras y lobos de penumbra;

Pero no da sonidos, ni cristales, ni fuego,

Sino plazas y plazas y otras plazas sin muros. (ver 158)

L'esprit de révolte et de subversion sous-jacent aux arts des avant-gardes concerne ici davantage la langue que l'attitude face à la mort. Cet esprit s'inscrit au cour de la syntaxe à partir de ruptures, d'écarts et d'images qui peuvent paraître, ou bien qui sont, parfois arbitraires. Il est également dans ce souffle lyrique qui s'enracine dans une tradition ancienne mais aussi déborde et dépasse les préceptes de la poétique surréaliste qui tend vers la liberté onirique des substitutions, des déplacements et des condensations imaginaires. Ce dépassement est précisément dans la tension permanente entre une poussée, une impulsion créative débridée et effrénée et une volonté de canaliser l'énergie dans des rails expressifs qui la domptent et lui offrent un cadre où chaque image brillera avec davantage d'intensité. De même, Lorca ne proteste pas contre la mort de son ami, contre son absurdité ou contre l'injustice des cieux face à la jeunesse foudroyée. La mort est ici envisagée dans sa dimension anthropologique la plus universelle, elle est

12 Eutimio Martín, 1988, Federico García Lorca, Antología comentada (I, Poesía), Madrid, Ediciones de la torre. 
fondement du tragique de la condition humaine et le poète la transfigure et la transcende en lui donnant une beauté convulsive et mythique.

La métaphore de la clarté de l'être revient à la dernière strophe pour substituer - semble dire Lorca à son ami - à l'absence de ton âme la présence de ton être qui s'incarne dans mes mots et les illumine. Ta présence éternelle est donc dans ces "palabras que gimen» ces mots, mes mots qui gémissent, dans ce LLANTO que l'on peut considérer comme un planctus avant-gardiste, qui pratique l'intertexte et le mélange des genres avant la lettre, et qui donne son titre à ce chant élégiaque pour Ignacio Sánchez Mejías.

\section{Bibliographie}

Correa G., 1975, La poesía mítica de Federico García Lorca, Madrid, Gredos.

Chevalier J. et Gheerbrant A., 1997, Dictionnaire des symboles, Paris, Laffont.

Del Río, A., 1952, Vida y obras de Federico García Lorca, Heraldo de Aragón, Zaragoza.

García Posada, M., 1979, Federico García Lorca, Madrid, escritores de todos los tiempos, EDAF.

García Lorca Federico, 1980, Obras Completas, tomo II, Madrid, Aguilar.

García Lorca Federico, 1990, Euvres complètes, tome II, André Belamich éd., Paris, Bibliothèque de la Pléiade $\mathrm{n}^{\circ} 369$.

García Lorca, Francisco, 1980, Federico y su mundo, Madrid, Alianza Editorial.

Martín E., 1988, Federico García Lorca, Antología comentada (I, Poesía), Madrid, Ediciones de la torre. 